\title{
Prognostic effects of the expression of inhibitor of DNA-binding family members on patients with lung adenocarcinoma
}

\author{
XIAOMIN LU ${ }^{1,2 *}$, LILI SHAO ${ }^{1,3^{*}}$, YE QIAN $^{2}$, YAN ZHANG $^{2}$, \\ YONGSHENG WANG $^{4}$, LIYUN MIAO ${ }^{4}$ and ZHIXIANG ZHUANG ${ }^{1}$ \\ ${ }^{1}$ Department of Oncology, The Second Affiliated Hospital of Soochow University, Suzhou, Jiangsu 215004; \\ ${ }^{2}$ Department of Oncology, Affiliated Haian Hospital of Nantong University, Nantong, Jiangsu 226601; \\ ${ }^{3}$ Department of Medical Oncology, Nantong University Affiliated Tumor Hospital, Nantong, \\ Jiangsu 226361; ${ }^{4}$ Department of Respiratory Diseases, Nanjing Drum Tower Hospital, \\ Medical School of Nanjing University, Nanjing, Jiangsu 210008, P.R. China
}

Received July 14, 2019; Accepted February 4, 2020

DOI: $10.3892 / \mathrm{ol} .2020 .12004$

\begin{abstract}
The basic helix-loop-helix (bHLH) transcription factors are negatively regulated by inhibitor of DNA-binding (ID) proteins. Several studies have demonstrated that ID family proteins are dysregulated in a variety of cancer types, including in lung adenocarcinoma (LUAD). In current study, the prognostic value of ID family members was evaluated by investigating publicly accessible databases, including Oncomine, Kaplan-Meier plotter, UALCAN and the Human Protein Atlas. It was observed that the mRNA expression of all ID members was downregulated in LUAD tumor tissues compared with those in normal tissues according to the Oncomine and UALCAN databases. Additionally, increased mRNA expression levels of ID2 and ID1 were associated with improved and poorer survival time, respectively. Notably, ID3 and ID4 expression was not associated with survival in patients with LUAD. At the protein level, high ID2 significantly predicted an improved survival outcome while high ID1 is associated with shorter survival time. Thus, the results indicate that the ID proteins, particularly ID2, exhibit significant prognostic value in LUAD. More studies are required to
\end{abstract}

Correspondence to: Dr Liyun Miao, Department of Respiratory Diseases, Nanjing Drum Tower Hospital, Medical School of Nanjing University, 1 Zhongshan Road, Nanjing, Jiangsu 210008, P.R. China E-mail: liyunmiao462@163.com

Professor Zhixiang Zhuang, Department of Oncology, The Second Affiliated Hospital of Soochow University, 1055 San Xiang Road, Suzhou, Jiangsu 215004, P.R. China

E-mail: 13951106391@139.com

*Contributed equally

Key words: inhibitor of DNA binding protein family members, prognosis, lung adenocarcinoma elucidate the underlying molecular mechanisms behind the role of the ID family in the development of LUAD.

\section{Introduction}

Lung cancer represents one of the leading causes of cancer-associated mortality, both globally as well as within China $(1,2)$. Non-small cell lung cancer (NSCLC) accounts for $80-85 \%$ of lung cancer cases, the majority of which are lung adenocarcinoma (LUAD) (3). Despite advances in the prevention, diagnosis and treatment of LUAD, the 5-year overall survival (OS) rate is <15\% (4). There have been numerous studies investigating LUAD prognosis-associated factors, including age, sex, tumor stage and certain genetic mutations [such as epidermal growth factor receptor (EGFR)], which may help to predict the OS; however, effective monitoring of certain genetic mutations is yet to be achieved, as they are either invasive or costly $(5,6)$. Therefore, the identification of more economical and convenient biomarkers able to predict OS time in patients with LUAD is imperative to facilitate efficient management and treatment of the disease.

The inhibitor of DNA binding (ID) proteins is a class of helix-loop-helix (HLH) transcription regulatory factors, including ID1, ID2, ID3 and ID4 (7). These proteins have been demonstrated to serve crucial roles in a wide range of tumor-associated processes, such as tumorigenesis, cell differentiation, cell cycle, migration and invasion, angiogenesis and metastasis $(8,9)$. Aberrant expression of the ID proteins has been revealed in a variety of human malignancies, including LUAD (10) and breast cancer $(11,12)$. However, the association between the expression of IDs and LUAD prognosis is yet to be elucidated. In the present study, compared with other malignancies, IDs, including ID1, ID2, ID3 and ID4, were universally downregulated in LUAD samples. Notably, cigarette smoke contains a complex mixture of $>6000$ chemicals, thus may alter gene expression and contribute to smoking-associated disease such as lung cancer. Thus, the association between IDs expression and smoking status was also analyzed in LUAD here. 
Notably, to the best of our knowledge, the prognostic value of IDs expression in predicting OS time in patients with LUAD has not yet been investigated. Therefore, the purpose of the present study was to summarize and update the currently available evidence from openly accessible databases regarding the association between the expression of various IDs and OS time in patients with LUAD.

\section{Materials and methods}

Oncomine analysis. The expression level of all ID proteins was analyzed in different types of human malignancies using a free-access public online database, Oncomine (www.oncomine.org), which contains a large amount of cancer microarray information. The paired Student's t-test was used to compare cancer vs. normal tissues and a fold-change of 2 with a P-value of $<0.01$ was considered to represent a clinically significance difference in expression, as in a previous study (13). Gene rank was analyzed by the percentile of the target gene in the top of all genes(top 10\%) measured in each study. The cell color was determined by the gene rank percentile for the analyses within the cell as follow: Dark blue, 100\%; blue, 50\%; light blue $10 \%$; dark red, $100 \%$, red, $50 \%$; and light red, $10 \%$.

UCLAN database analysis. The open access database UCLAN (http://ualcan.path.uab.edu/analysis.html) was used to analyze the association between the expression levels of various IDs and Tumor-Node-Metastasis (TNM) staging and smoking status of patients with LUAD (14). This database provides easy access to the publicly available cancer OMICS information from The Cancer Genome Atlas (TCGA) and the MET500 (a database comprised of whole-exome and -transcriptome sequencing of 500 adult patients with metastatic solid tumors of diverse lineage and biopsy sites), allowing users to identify biomarkers or perform in silico validation of potential genes of interest. The median expression value was used as cut-off and significant difference between groups was assessed using the unpaired two-sample Student's t-test.

Human protein atlas. The HPA (https://www.proteinatlas. org/) is an open access program that maps all human proteins in cells and tissues (15). The Pathology Atlas of the HPA depicts the association between specific protein expression levels and the OS time of the patients with LUAD (16). Here the HPA was used to analyze the relationship between IDs protein expression and survival time.

Kaplan-Meier plotter survival analysis. To analyze the prognostic values of ID expression levels in LUAD samples, the Kaplan-Meier plotter (www.kmplot.com) was used. This demonstrated the association between the OS time of patients with LUAD with high- or low-ID mRNA expression based on median expression as cut-off value. The Log-rank was used to compare the difference between curves and is presented on the webpage (17).

Statistical analysis. All data are presented as the mean \pm standard deviation (SD). All statistical analyses were performed using one-way ANOVA or unpaired two sample t test (SPSS 22.0; IBM Corp.). The survival curves were calculated using the Kaplan-Meier method and statistically compared using a log-rank test. Differences were considered statistically significant when the P-values were $<0.05$, unless otherwise specified.

\section{Results}

mRNA expression levels of ID family members in human lung cancer tissues. ID proteins (ID1, ID2, ID3 and ID4) exhibited varied expression patterns in tumors and normal tissues, as well as in other human malignancies. The difference in expression levels was analyzed using the online tumor database Oncomine, which had a total of 445, 457, 420 and 442 analyses describing ID1, ID2, ID3 and ID4 expression, respectively. Regarding lung cancer, 8 studies reported that ID1 was significantly downregulated compared with normal tissues. Of these, 6 studies revealed that ID1 was significantly downregulated in LUAD compared with the normal tissues. Regarding ID2, 5 studies revealed that it was significantly downregulated in lung cancer and, of these, 4 studies described the significant downregulation of ID2 in LUAD tissues. ID3 was revealed to be significantly downregulated in 4 studies of LUAD tissues. Moreover, ID4 was demonstrated to be downregulated in 9 studies on lung cancer, 7 of which were specifically describing LUAD tissues compared with normal tissues (Fig. 1).

Downregulation of ID mRNA levels according to UCLAN database. To further explore the expression of ID proteins in LUAD, data was analyzed using the UCLAN database and it was observed that the entire ID family, comprising ID1, ID2, ID3 and ID4, were all significantly downregulated in LUAD compared with that in normal tissues (Fig. 2).

Downregulation of the ID family according to TCGA database of different clinical features. ID family expression was further analyzed between different stages of LUAD and it was observed that all ID proteins were downregulated between stage I and IV in LUAD compared with those in the normal tissues (Fig. 3A-D). Moreover, smoking status did not significantly influence expression levels, as IDs expressions were significantly downregulated in LUAD compared with that in the normal tissues, irrespective of the smoking history (Fig. 3E-H). The current data indicate that IDs serve an important role in the tumorigenesis rather than in progression of LUAD, and that their expression is not significantly associated with tobacco usage.

Dysregulation of ID1 and ID2 mRNA expression, and its association with OS time. Subsequently, data from the KM plotter was analyzed to identify whether members of this family are associated with the survival time of patients with LUAD. It was observed that higher expression of ID1 was associated with poor survival in LUAD ( $\mathrm{P}<0.05$ vs. $\mathrm{P}=0.0083$; Fig. 4A). However, higher ID2 mRNA expression was significantly associated with a longer OS time in LUAD ( $\mathrm{P}<0.05$; Fig. 4B). Notably, regarding ID3 and ID4, there was no significant difference in OS time between the high- and low-expression group, with the median expression used as the cut-off value (both P>0.05; Fig. 4C and D). 


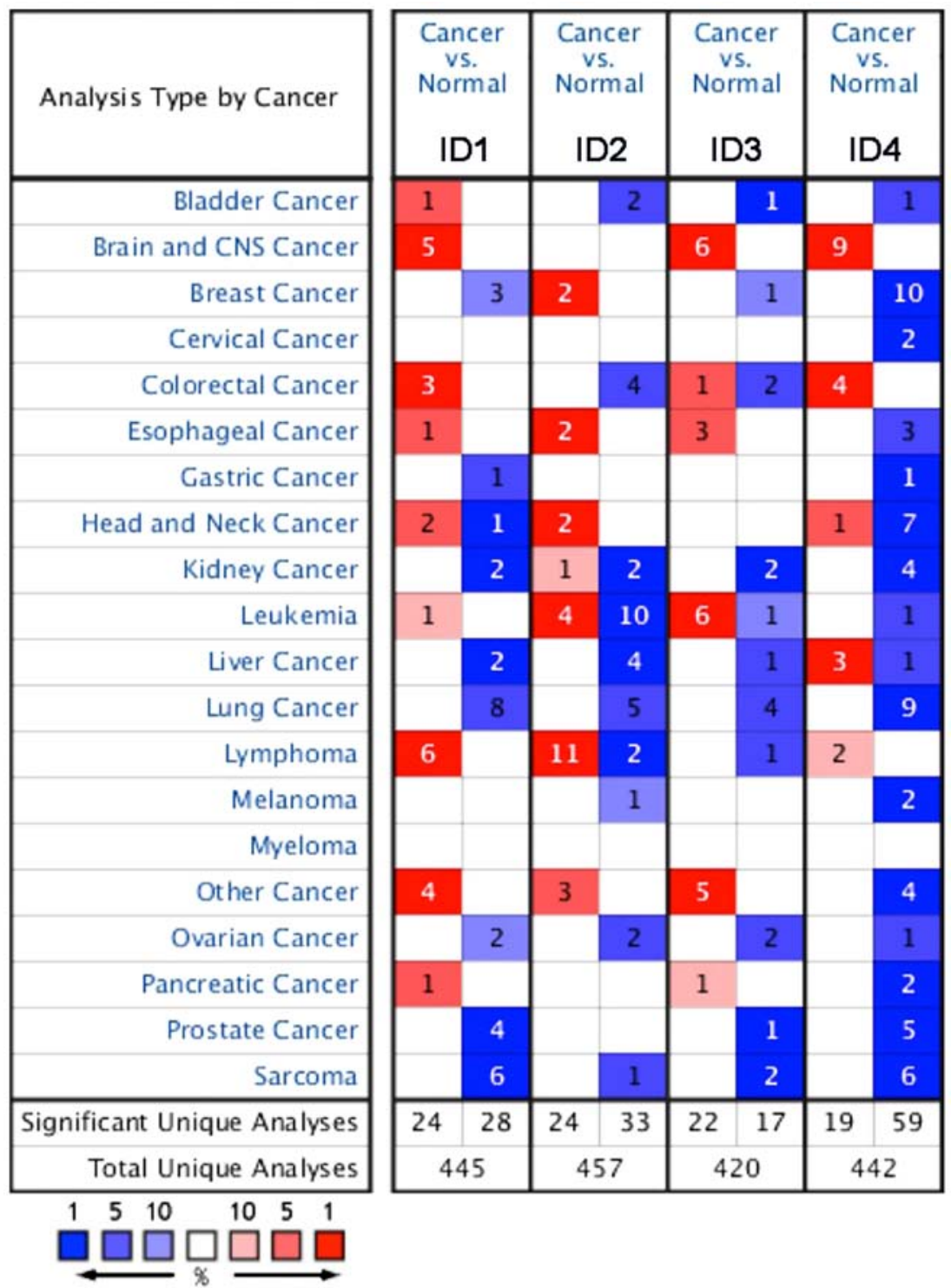

Cell color is determined by the best gene rank percentile for the analyses within the cell.

NOTE: An analysis may be counted in more than one cancer type.

Figure 1. mRNA expression patterns of IDs in different types of human malignancy. mRNA expression of ID family members (cancer vs. normal tissue) was analyzed with the Oncomine database. Red indicates high expression and blue indicates low expression of the target gene. The P-value threshold was maintained to be 0.01 . The number in each cell represents the number of analyses that meets this threshold. Gene rank was analyzed by the percentile of the target gene in the top of all genes measured in each study. The cell color was determined by the gene rank percentile for the analyses within the cell. $\mathrm{P}<0.01$ and fold-change $>2$ were used to establish the threshold. ID, inhibitor of DNA binding; CNS, central nervous system.

Upregulation of ID1 and ID2 protein expression is associated with $O S$ time in patients with LUAD. Data from the HPA database were analyzed to discover whether there was an association between level of protein expression and OS time. It was observed that increased ID2 protein expression was positively associated with improved survival time while high ID1 protein was associated with poor survival time in patients with LUAD (Fig. 5A and $\mathrm{B} ; \mathrm{P}<0.05$ ). However, the protein expression levels of ID3 and ID4 did not have any significant influence on the OS time of the patients with LUAD ( $\mathrm{P}=0.077$, Fig. 5C; $\mathrm{P}=0.18$, Fig. 5D).

\section{Discussion}

LUAD treatment modality has been revolutionized by the implementation of targeted therapy against genes such as
EGFR and ALK, which has improved clinical outcomes significantly $(18,19)$. Other targeted treatments include those against more uncommon mutations such as BRAF V600E and ROS1 (20,21). Immunotherapy can to be used alone or in combination with chemotherapy to treat LUAD as the first-line therapeutic option, depending on the expression level of PD-L1 (22-24). This paradigm shift in targeted treatment options is based on the underlying mechanisms of LUAD progression, such as the EGFR signaling pathway and ALK translocations.

IDs are a family of transcription regulators containing a highly conserved HLH domain. These proteins influence a variety of tumor processes, including tumorigenesis, progression, tumor differentiation, cell cycle progression and metastasis (8). Moreover, IDs are the downstream targets 

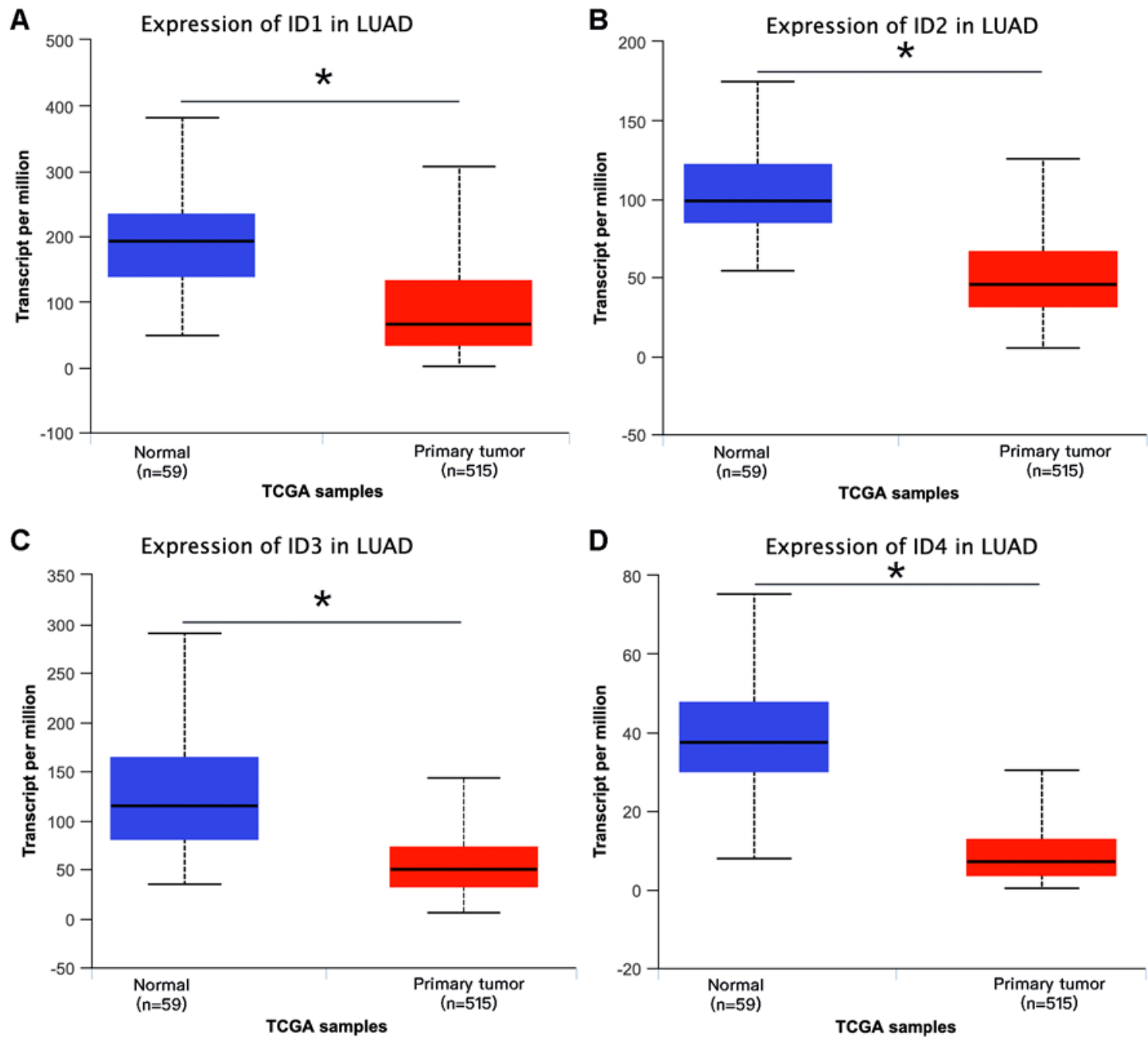

Figure 2. mRNA expression of the ID family members in LUAD and normal tissues from the TCGA database. mRNA expression levels of (A) ID1, (B) ID2, (C) ID3 and (D) ID4 in LUAD compared with those in normal tissues. Significant difference between groups was assessed using the unpaired two-sample Student's t-test. The two comparison groups are normal vs. primary tumor. All data are presented as the mean \pm standard deviation (SD). All groups are different groups of patients or normal controls. " $\mathrm{P}<0.05$. ID, inhibitor of DNA binding; TCGA, The Cancer Genome Atlas; LUAD, lung adenocarcinoma.

of a number of oncogenic pathways, making them potential targets for cancer therapeutics (25). However, from the known literature, the different ID members have contradictory roles in distinct types of cancer, and the role of IDs in LUAD is yet to be elucidated. In the present study, the mRNA expression of the ID family members was identified using the Oncomine database. It was revealed that IDs were differentially expressed between different types of human malignancies. In lung cancer, all ID family members were significantly downregulated. Notably, IDs were decreased mostly when observing the histology of the LUAD compared with that in the normal tissues. To confirm the aforementioned results, data from UCLAN (a large accessible database containing data from TCGA and MET500) were investigated. It was revealed that all IDs were significantly downregulated in LUAD tissues compared with those in normal tissues, irrespective of stage or smoking history, indicating their role in tumorigenesis and tumor progression. Castañon et al (26) reported that ID1 is upregulated in NSCLC tissues and that this is associated with shorter OS time. ID family members may influence cell fate determination, differentiation and cell proliferation by binding to bHLH. However, each individual ID has diverse role in these processes. It was found that ID1, ID3 and ID4 were co-expressed in LUAD. Moreover, in the study by Castañon et al (26), the expression levels of ID1 and ID3 were demonstrated to be associated with each other, and were also associated with a poor clinical outcome in patients with locally advanced NSCLC treated with definitive chemoradiotherapy. In the present study, it was revealed that all ID family members were downregulated in LUAD, irrespective of stage or smoking habits. However, to understand the distinct mechanisms underlying the roles of the different IDs in LUAD progression, further research is needed.

It has been reported that in methylated glioblastomas, ID4 mRNA is significantly reduced and ID4 methylation is significantly associated with a favorable clinical outcome (27). Other studies reported that an increased expression level of ID4 along with a concomitant loss of the BRCA proteins in triple-negative breast cancer adversely affect the survival outcome of affected patients (28). However, it was revealed that higher ID2 mRNA expression predicted an improved survival outcome, while the expression levels of both ID3 and 
A Expression of ID1 in LUAD based on individual cancer stages

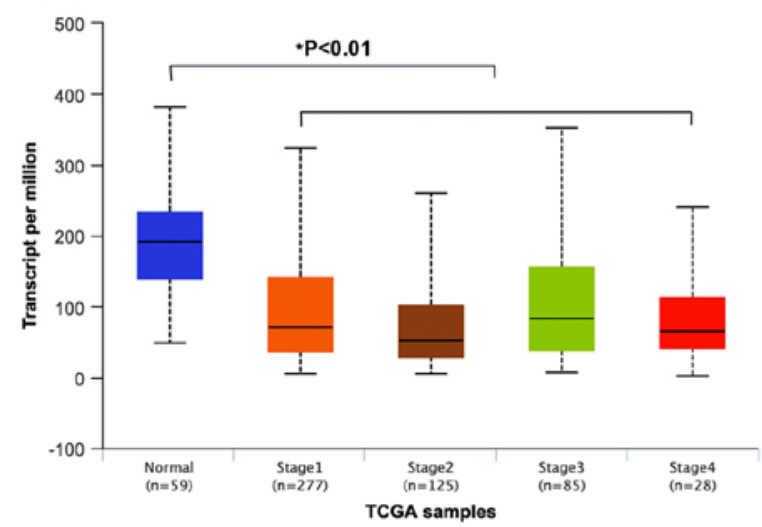

C Expression of ID3 in LUAD based on individual cancer stages

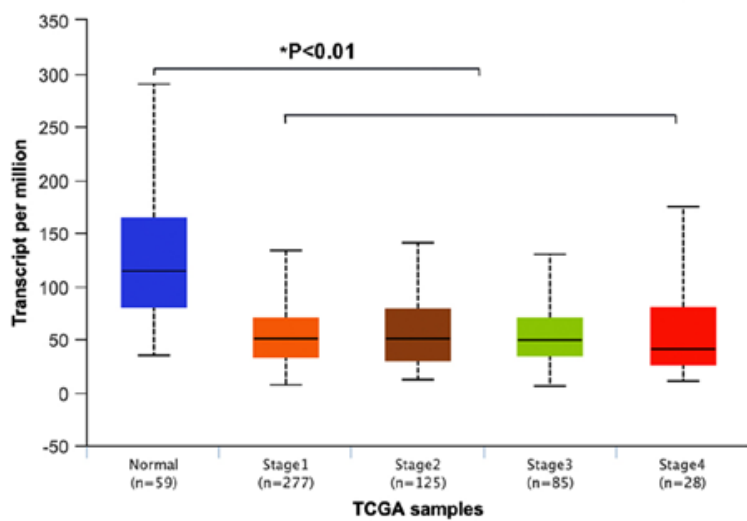

E Expression of ID1 in LUAD based on patient's smoking habits

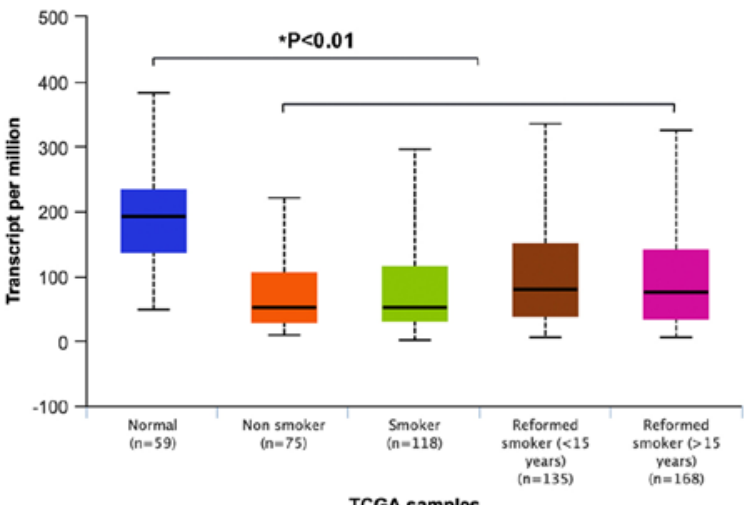

TCGA samples

$\mathbf{G}$ Expression of ID3 in LUAD based on patient's smoking habits

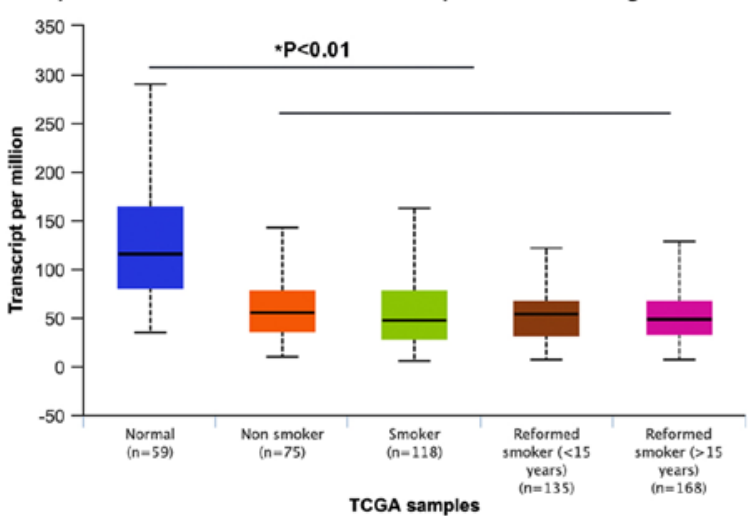

B Expression of ID2 in LUAD based on individual cancer stages

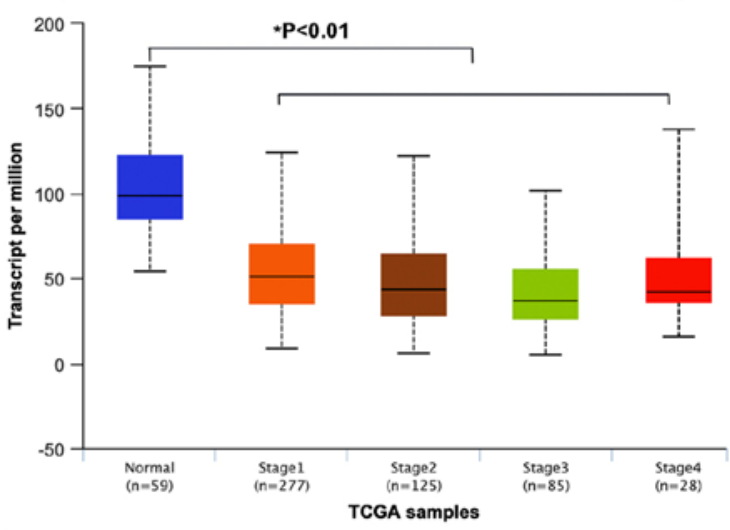

D Expression of ID4 in LUAD based on individual cancer stages

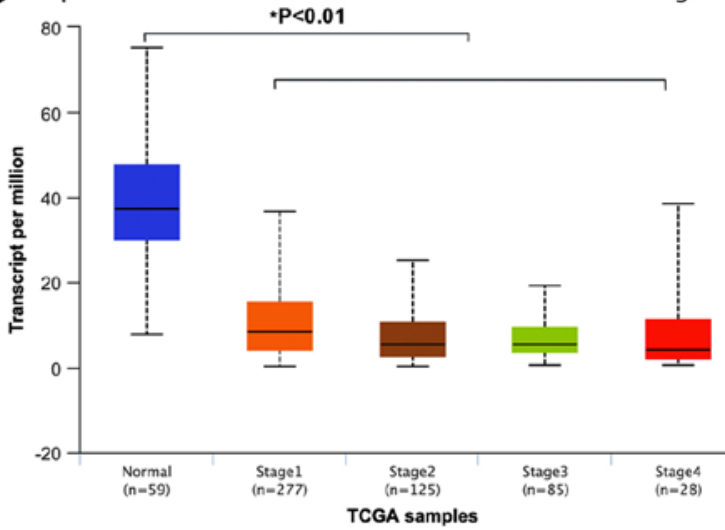

F Expression of ID2 in LUAD based on patient's smoking habits

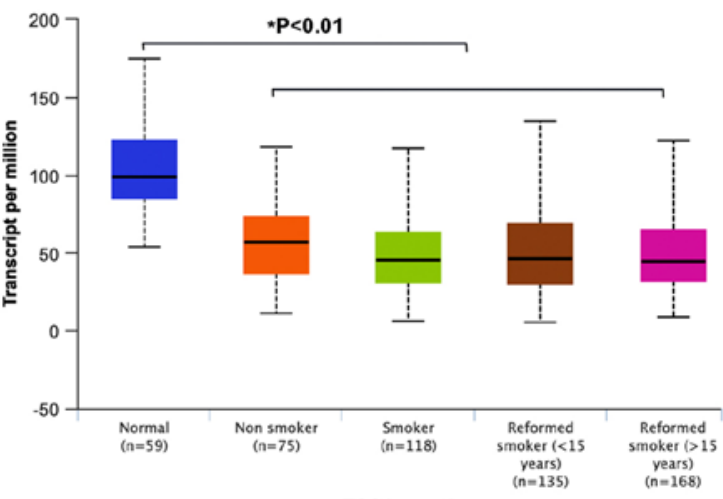

TCGA samples

H Expression of ID4 in LUAD based on patient's smoking habits

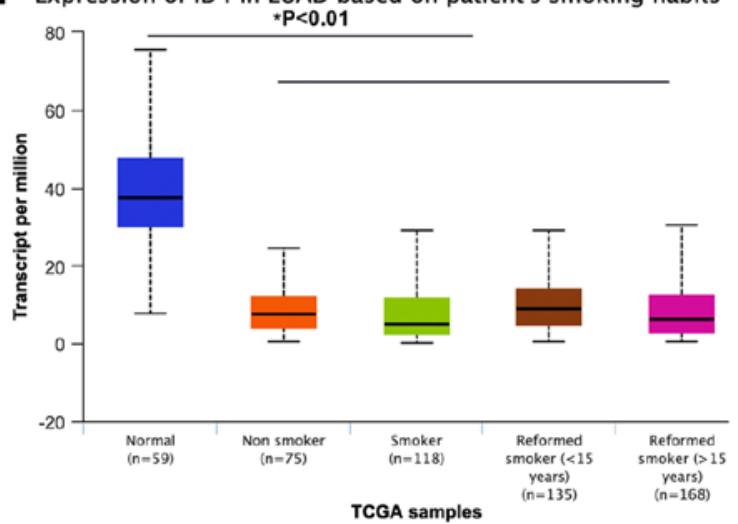

Figure 3. Association between the gene expression levels of ID family proteins with cancer stage or smoking status, using data from TCGA. mRNA expression levels of (A) ID1, (B) ID2, (C) ID3 and (D) ID4 in normal tissues compared with those in tissues at various different cancer stages. (E) ID1, (F) ID2, (G) ID3 and (H) ID4 mRNA expression levels in normal tissues normalized to patient status. Data are presented as the mean \pm standard deviation (SD). One-way ANOVA was used to test differences among groups. If statistical significance was obtained through ANOVA ( $\mathrm{P}<0.05)$, Tukey's post-hoc test was used to make multiple comparisons. $\mathrm{P}<0.05$ was considered to indicate a statistically significant difference. ID, inhibitor of DNA binding; TCGA, The Cancer Genome Atlas; LUAD, lung adenocarcinoma. 

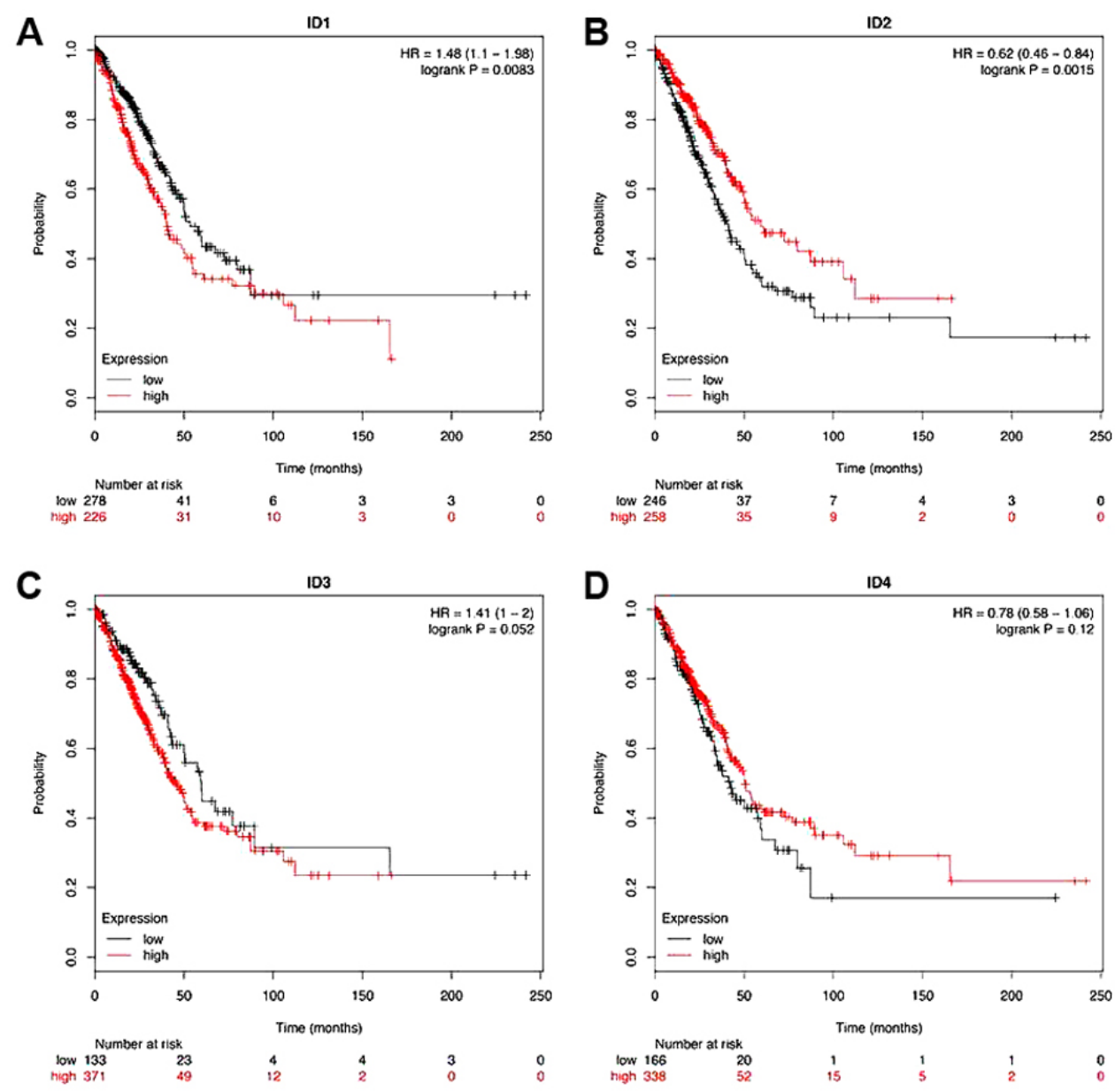

Figure 4. Genetic analysis of the prognostic values of the ID family members in patients with LUAD. Kaplan-Meier overall survival time estimates for (A) ID1, (B) ID2, (C) ID3 and (D) ID4 mRNA levels. P<0.05 was considered to indicate a statistically significant difference. ID, inhibitor of DNA binding; LUAD, lung adenocarcinoma; HR, hazard ratio.
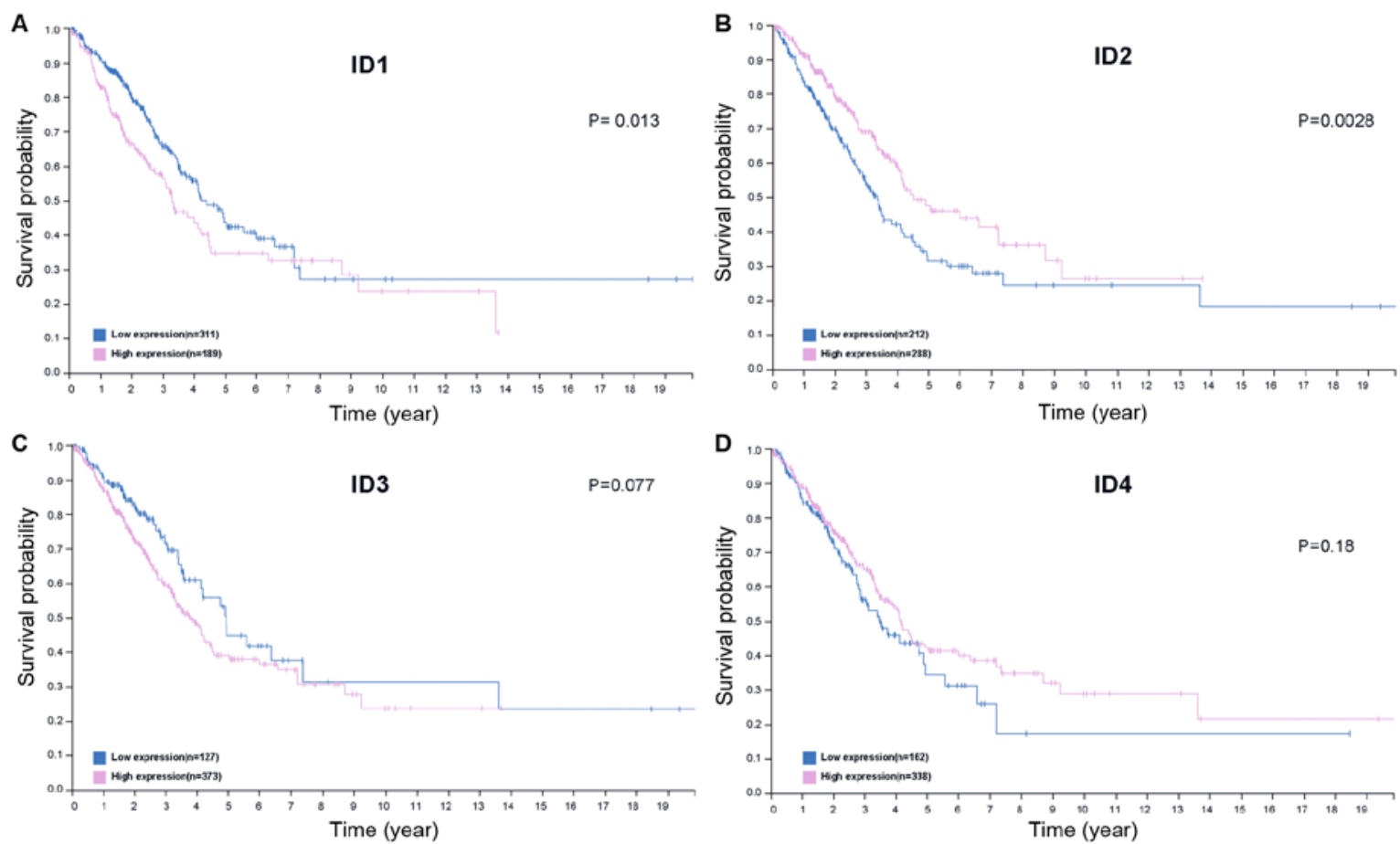

Figure 5. Proteomic analysis of the prognostic values of the ID family members in LUAD. Kaplan-Meier overall survival estimates for (A) ID1, (B) ID2, (C) ID3 and (D) ID4 protein levels. The Log-rank was used to compare the difference between survival time. P $<0.05$ was considered to indicate a statistically significant difference. ID, inhibitor of DNA binding; TCGA, The Cancer Genome Atlas; LUAD, lung adenocarcinoma. 
ID4 mRNA did not exert any significant effect on clinical outcome. Notably, ID1 was downregulated in LUAD and the high mRNA expression group was significantly associated with a poorer survival time in patients with LUAD. Thus, contradictory results were obtained, which should be validated on tumor samples. At the protein level, it was revealed that high ID2 expression was associated with a longer OS time, while high ID1 was associated with a shorter survival time. Thus, the expression of ID1 and survival time is contradictory and should be validated on tumor samples. Therefore, it was supposed that amongst the ID family members, ID2 may serve a critical role in the genesis and progression of LUAD, thereby having a significant clinical outcome favoring survival of the patients with LUAD.

Wen et al (12) revealed that ID2 upregulated notch3 expression via blocking the binding of E2A to an E-box motif in the notch 3 promoter; further decreased expression of ID2 was associated with worse long-term metastasis-free survival in patients with breast cancer (12). Moreover, Bae et al (29) revealed that ID2 expression was significantly higher in the head and neck squamous cell carcinoma (HNSCC) cells with stemness, compared with that in differentiated HNSCC cells; furthermore, increased expression of ID2 was closely associated with poorer post-treatment survival rates in patients with HNSCC. By contrast, it was observed in the current study that increased expression of ID2 was strongly associated with better survival outcomes in patients with LUAD. These differential findings indicate that different types of human cancer have varied ID2 expression patterns.

Several potential limitations of the present study should be noted; although it was demonstrated that IDs proteins serve an important role in LUAD and may represent a therapeutic target, all data were retrieved from public databases and not validated using tumor and normal samples. In addition, Gene Ontology and Kyoto Encyclopedia of Genes and Genomes pathway analyses were not performed to identify genes associated with IDs in LUAD, as the current study was focused on determining the role of the ID family in the progression of LUAD. In the present study, it was revealed that the mRNA expression of ID1 and ID2 was associated with survival outcomes, and at the protein level, ID1 and ID2 was associated with OS time in patients with LUAD. However, based on the contradictory results of ID1, it was proposed that ID2 may serve a critical role in the genesis and progression of LUAD. To further elucidate the underlying molecular mechanism behind the role of ID2 in LUAD, more in-depth studies are warranted to investigate the exact functions of ID2 in tumorigenesis and the development of LUAD.

\section{Acknowledgements}

Not applicable.

\section{Funding}

No funding was received.

\section{Availability of data and materials}

The datasets generated and/or analyzed during the present study are available in the Oncomine (https://www.oncomine. org), UCLAN (http://ualcan.path.uab.edu/analysis.html), Kaplan Meier-plotter (kmplot.com/analysis), and Human Protein Atlas (https://www.proteinatlas.org).

\section{Authors' contributions}

$\mathrm{XL}, \mathrm{LM}$ and $\mathrm{ZZ}$ participated in the design of the study, data acquisition and analysis as well as drafting the manuscript. LS and YQ were responsible for the data analysis. YZ and YW participated in data acquisition, analysis, and interpretation. All authors read and approved the final manuscript.

\section{Ethics approval and consent to participate}

Not applicable.

\section{Patient consent for publication}

Not applicable.

\section{Competing interests}

The authors declare that they have no competing interests.

\section{References}

1. Bray F, Ferlay J, Soerjomataram I, Siegel RL, Torre LA and Jemal A: Global cancer statistics 2018: GLOBOCAN estimates of incidence and mortality worldwide for 36 cancers in 185 countries. CA Cancer J Clin 68: 394-424, 2018.

2. Chen W, Zheng R, Baade PD, Zhang S, Zeng H, Bray F, Jemal A, $\mathrm{Yu}$ XQ and He J: Cancer statistics in China, 2015. CA Cancer J Clin 66: 115-132, 2016.

3. Cancer Genome Atlas Research Network: Comprehensive molecular profiling of lung adenocarcinoma. Nature 511: 543-550, 2014.

4. Johnson DH, Schiller JH and Bunn PA Jr: Recent clinical advances in lung cancer management. J Clin Oncol 32: 973-982, 2014.

5. Li P, Gao Q, Jiang X, Zhan Z, Yan Q, Li Z and Huang C: Comparison of clinicopathological features and prognosis between ALK rearrangements and EGFR mutations in surgically resected early-stage lung adenocarcinoma. J Cancer 10: 61-71, 2019.

6. Paesmans M: Prognostic and predictive factors for lung cancer. Breathe 9: 112-121, 2012.

7. Ke J, Wu R, Chen Y and Abba ML: Inhibitor of DNA binding proteins: Implications in human cancer progression and metastasis. Am J Transl Res 10: 3887-3910, 2018.

8. Sikder HA, Devlin MK, Dunlap S, Ryu B and Alani RM: Id proteins in cell growth and tumorigenesis. Cancer Cell 3: 525-530, 2003 .

9. Yokota Y: Id and development. Oncogene 20: 8290-8298, 2001.

10. Antonângelo L, Tuma T, Fabro A, Acencio M, Terra R, Parra E, Vargas F, Takagaki T and Capelozzi V: Id-1, Id-2, and Id-3 co-expression correlates with prognosis in stage I and II lung adenocarcinomapatients treated with surgery and adjuvant chemotherapy. Exp Biol Med (Maywood) 241: 1159-1168, 2016.

11. Zhou XL, Zeng, Ye YH, Sun SM, Lu XF, Liang WQ, Chen CF and Lin HY: Prognostic values of the inhibitor of DNA-binding family members in breast cancer. Oncol Rep 40: 1897-1906, 2018.

12. Wen XF, Chen M, Wu Y, Chen MN, Glogowska A, Klonisch T and Zhang GJ: Inhibitor of DNA binding 2 inhibits epithelial-mesenchymal transition via upregulation of Notch3 in breast cancer. Transl Oncol 11: 1259-1270, 2018.

13. Lin HY, Zeng, Liang YK, Wei XL and Chen CF: GATA3 and TRPS1 are distinct biomarkers and prognostic factors in breast cancer: Database mining for GATA family members in malignancies. Oncotarget 8: 34750-34761, 2017. 
14. Chandrashekar DS, Bashel B, Balasubramanya SAH Creighton CJ, Ponce-Rodriguez I, Chakravarthi BVSK and Varambally S: UALCAN: A portal for facilitating tumor subgroup gene expression and survival analyses. Neoplasia 19: 649-658, 2017.

15. Thul PJ, Åkesson L, Wiking M, Mahdessian D, Geladaki A, Ait Blal H, Alm T, Asplund A, Björk L, Breckels LM, et al: A subcellular map of the human proteome. Science 356: eaal3321, 2017.

16. Uhlén M, Fagerberg L, Hallström BM, Lindskog C, Oksvold P, Mardinoglu A, Sivertsson Å, Kampf C, Sjöstedt E, Asplund A, et al: Proteomics. Tissue-based map of the human proteome. Science 347: 1260419, 2015.

17. Nagy A, Lánczky A, Menyhárt O and Győrffy B: Validation of miRNA prognostic power in hepatocellular carcinoma using expression data of independent datasets. Sci Rep 8: 11515, 2018.

18. Zhou C, Wu YL, Chen G, Feng J, Liu XQ, Wang C, Zhang S, Wang J, Zhou S, Ren S, et al: Erlotinib versus chemotherapy as first-line treatment for patients with advanced EGFR mutation-positive non-small-cell lung cancer (OPTIMAL, CTONG-0802): A multicentre, openlabel, randomised, phase 3 study. Lancet Oncol 12: 735-742, 2011.

19. Solomon BJ, Mok T, Kim DW, Wu YL, Nakagawa K, Mekhail T, Felip E, Cappuzzo F, Paolini J, Usari T, et al: First-line crizotinib versus chemotherapy in ALK-positive lung cancer. N Engl J Med 371: 2167-2177, 2014.

20. Shaw AT and Solomon BJ: Crizotinib in ROS1-rearranged non-small-cell lung cancer. N Engl J Med 372: 683-684, 2015.

21. Planchard D, Smit EF, Groen HJM, Mazieres J, Besse B, Helland A, Giannone V, D'Amelio AM Jr, Zhang P, Mookerjee B and Johnson BE: Dabrafenib plus trametinib in patients with previously untreated $\mathrm{BRAF}^{\mathrm{V} 600 \mathrm{E}}$-mutant metastatic non-small-cell lung cancer: An open-label, phase 2 trial. Lancet Oncol 18: 1307-1316, 2017.

22. Reck M, Rodríguez-Abreu D, Robinson AG, Hui R, Csôszi T, Fülöp A, Gottfried M, Peled N, Tafreshi A, Cuffe S, et al: Pembrolizumab versus Chemotherapy for PD-L1-Positive Non-Small-Cell Lung Cancer. N Engl J Med 375: 1823-1833, 2016.
23. Mok TSK, Wu YL, Kudaba I, Kowalski DM, Cho BC, Turna HZ, Castro G Jr, Srimuninnimit V, Laktionov KK, Bondarenko I, et al: Pembrolizumab versus chemotherapy for previously untreated, PD-L1-expressing, locally advanced or metastatic non-small-cell lung cancer (KEYNOTE-042): A randomised, open-label, controlled, phase 3 trial. Lancet 393: 1819-1830, 2019.

24. Gandhi L, Rodríguez-Abreu D, Gadgeel S, Esteban E, Felip E, De Angelis F, Domine M, Clingan P, Hochmair MJ, Powell SF, et al: Pembrolizumab plus chemotherapy in metastatic non-small-cell lung cancer. N Engl J Med 378: 2078-2092, 2018.

25. McAllister SD, Christian RT, Horowitz MP, Garcia A and Desprez PY Cannabidiol as a novel inhibitor of Id-1 gene expression in aggressive breast cancer cells. Mol Cancer Ther 6: 2921-2927, 2007.

26. Castañon E, Bosch-Barrera J, López I, Collado V, Moreno M, López-Picazo JM, Arbea L, Lozano MD, Calvo A and Gil-Bazo I: Id 1 and Id 3 co-expression correlates with clinical outcome in stage III-N2 non-small cell lung cancer patients treated with definitive chemoradiotherapy. J Transl Med 11: 13, 2013.

27. Martini M,Cenci T, D'Alessandris GQ, Cesarini V, Cocomazzi A, Ricci-Vitiani L, De Maria R, Pallini R and Larocca LM: Epigenetic silencing of Id4 identifies a glioblastoma subgroup with a better prognosis as a consequence of an inhibition of angiogenesis. Cancer 119: 1004-1012, 2013.

28. Thike AA, Tan PH, Ikeda M and Iqbal J: Increased ID4 expression, accompanied by mutant p53 accumulation and loss of BRCA $1 / 2$ proteins in triple-negative breast cancer, adversely affects survival. Histopathology 68: 702-712, 2016.

29. Bae WJ, Koo BS, Lee SH, Kim JM, Rho YS, Lim JY, Moon JH, Cho JH and Lim YC: Inhibitor of DNA binding 2 is a novel therapeutic target for stemness of head and neck squamouscell carcinoma. Br J Cancer 117: 1810-1818, 2017.

This work is licensed under a Creative Commons Attribution-NonCommercial-NoDerivatives 4.0 International (CC BY-NC-ND 4.0) License. 